Journal of Engineering and Applied Sciences 14 (8): 2661-2671, 2019

ISSN: 1816-949X

(C) Medwell Journals, 2019

\title{
Optimization Synthesis of the Car Body Stabilization System
}

\author{
Generalova Alexandra Alexandrovna and Khabibullin Rinat Rashidovich \\ Penza State University, Krasnaya Street 40, Penza, Russia
}

\begin{abstract}
One of the main directions for creating highly efficient automotive components is the formation of a set of their properties (parameters and characteristics) at the stage of the design solution that ensures satisfaction of the set of conflicting requirements of multi-mode operation conditions. In this study, the system for stabilizing the suspension of the car is proposed. The characteristics of the developed stabilization system, modeled in CAE SolidWorks are determined. The car stabilization system will increase the safety of management and comfort when driving a vehicle. In this study, an approach is proposed for determining the optimal values of structural elements of the system as well as calculating the spectrum near the optimal values of these elements. The study considers the optimization method in the mode of virtual simulation of parameters and characteristics of the car stabilization system in the absence of direct access to mathematical models of the process.
\end{abstract}

Key words: Car, stabilization, body, optimization, multicriteria, performance

\section{INTRODUCTION}

Structural optimization of technical systems is realized, basically, at a stage of formation of technical decisions. Its content is the construction of structures from known elements of the subject domain in question and the selection of the best by some criteria. In this case, the space-time layout of elements with the establishment of conjugations between them leads to the restriction of the degrees of freedom (properties) of individual elements and the appearance of new properties of a new design that are not inherent in each of the elements individually. Thus, if parametric optimization provides the desired increase in desired (necessary) design properties and/or characteristics of the process of its functioning, structural optimization provides the formation (synthesis) of a fundamentally new design property. The number of works devoted to the topic of optimization synthesis of car suspension is now great. The researchers Chun et al. (2007) consider the design optimization of suspension system by the criterion of reliability and by Suh and Yoon (2018) propose a comprehensive method of analysis for the optimization of the suspension characteristics with respect to design changes, chassis and performance strength of the modified design of the military vehicle. Sidel'nikov and Brom (2018) presents optimization models for element failures.

When moving a car in urban conditions, along the road and off-road, the body of the car is displaced and tilted under the action of inertial forces. Roll during turn, braking and during acceleration creates problems for the driver. With excessive moves and rolls, the suspension has a positive camber. This is especially noticeable in the McPherson type suspension where the angle of collapse is initially static and the camber changes with the body roll. Not so much this effect is manifested in multi-link suspension the car heels andthe tires are less prone to tilt relative to the canvas. This combination of circumstances, like roll and limited possibilities of suspension geometry, causes the tires to stand on the outer edge, resulting in a reduced contact spot and loss of grip (Bogatyryov et al., 2004; Pavlov and Kuvshinov 2011; Pavlov, 2014).

The purpose of the proposed development is to simplify the design and improve the performance of vehicles by compensating for unwanted vibrations of the body in the transverse plane.

\section{MATERIALS AND METHODS}

Modern $\mathrm{CAD} / \mathrm{CAE} / \mathrm{CAM}$ systems together are system design tools that provide a significant reduction in time and material costs in the process of creating vehicles of the required quality. During the formation of the product model, the designer has the opportunity to evaluate its properties (mass-dimensional parameters, strength, frequency characteristics, etc.) without going beyond the design. The standard of de facto 3D modeling of the SolidWorks system is characterized by the presence of many tools of such express analysis, one of which is the SolidWorks Simulation module.

To solve optimization problems, the following conditions must be met: formulate the initial problem on

Corresponding Author: Generalova Alexandra Alexandrovna, Penza State University, Krasnaya Street 40, Penza, Russia 
the basis of which the subsequent optimization is carried out (optimization can be carried out under complex conditions that reflect the properties of the product under study on the results of solving various problems from the permissible list), define the purpose of optimizing, define design variables for which limits should be explicitly set (by default, the program takes the initial value of the parameter as an average forming the limits of its change in the range from $0.5-1.5$ of the initial value), define restrictions on at least one product feature (restrictions may correspond to different types of tasks), specify an iterative process option that minimizes the number of intermediate calculations), balance the ranges of variation of product parameters (the program is likely to use planning at three levels with not all combinations of geometric parameters can be physically implemented. Otherwise, the solution will be stopped. Next, you should adjust the parameters and their change intervals which should again be explicitly specified), in solving the problem of complex optimization for the models of its particular problems should be used the same material.

\section{RESULTS AND DISCUSSION}

The task of the development is to ensure the smoothness of the stroke and energy intensity of the suspension, namely (D'yachkov et al., 2016a, b):

- Improvement of regression characteristics of the shock absorber

- Development of a dynamic suspension stabilization design with an upgraded shock absorber

The technical result achieved by the development is the implementation of the following technical solutions:

- Mechanism of dynamical change of the regressive characteristic of the shock absorber

- Dynamic suspension stabilization system

To achieve this technical result, the following design of stabilization of the transverse stability of the vehicle is proposed (Fig. 1). The system consists of: solenoid valve 1 , hydraulic cylinder 2 , stabilizer stand 3 , stabilizer 4 , connecting hoses 5 , stabilizer mounting 6 . The solenoid valve 1 is connected to the ECU of the car 7 by means of a cable train.

The hydraulic cylinders 2 and the stabilizer stand 3 are rigidly connected to the car body. The solenoid valve 2 bymeans of the ECU 7 forcibly opens or closes the fluid flow channels in the dynamic suspension system of

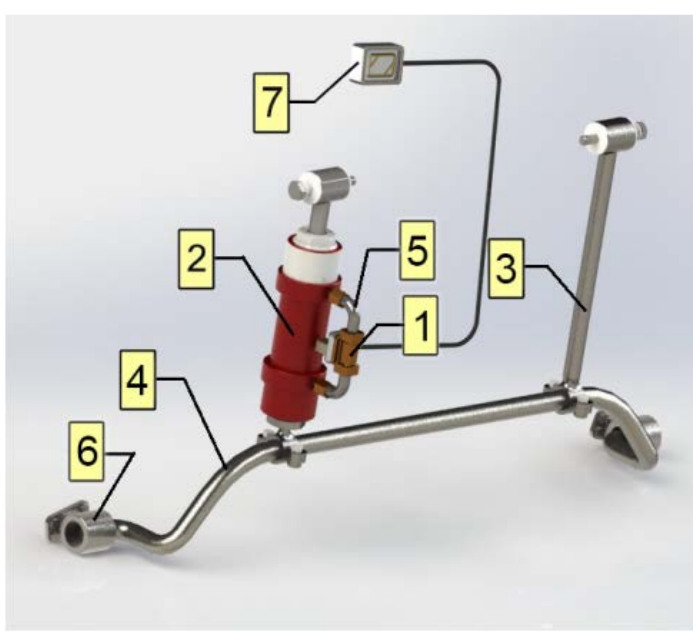

Fig. 1: The system of dynamic stabilization of the suspension: 1) Solenoid valve; 2) Hydraulic cylinder; 3) Stabilizer stand; 4) Stabilizer; 5) Connecting hoses; 6) Mounting the stabilizer and 7) $\mathrm{ECU}$

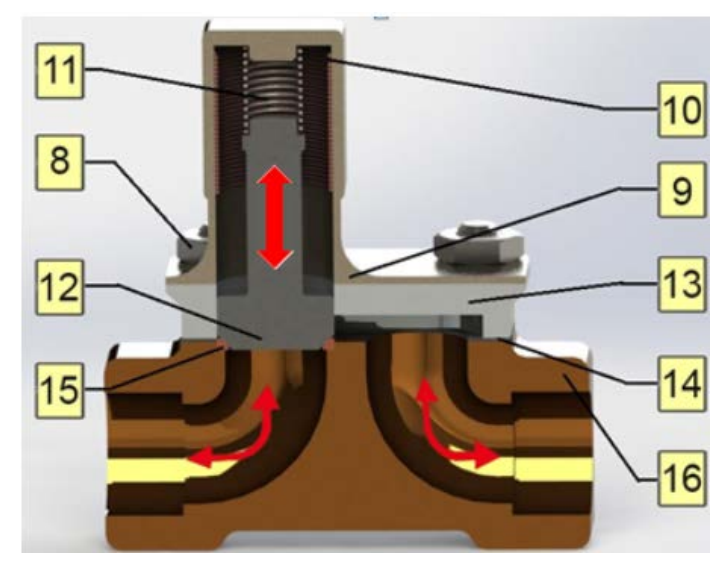

Fig. 2: Solenoid valve: 8) Nuts; 9) Cap; 10) Coil; 11) Spring; 12) Core; 13) Head, 14) Gasket; 15) O-ring and 16) Housing

the vehicle. The transducers for vertical and transverse accelerations must be installed on the vehicle, the signal from which is fed to ECU 7. The solenoid valve consists of nuts 8 , cover 9 , coils 10 , springs 11 , core 12 , head 13 , gaskets 14, O-ring 15, housing 16 (Fig. 2).

The stabilizer stand 3 is fixed to the stabilizer 4 by means of a flange 17 with two bolts 18 . To the car body the stand is fastened with a silent block 19 (Fig. 3).

The silent block 19 is installed in the stabilizer stand 3 and consists of a guide 20 , a sleeve 21 , a rubber insert 22 , a bolt 23 and a nut 24 (Fig. 4). 


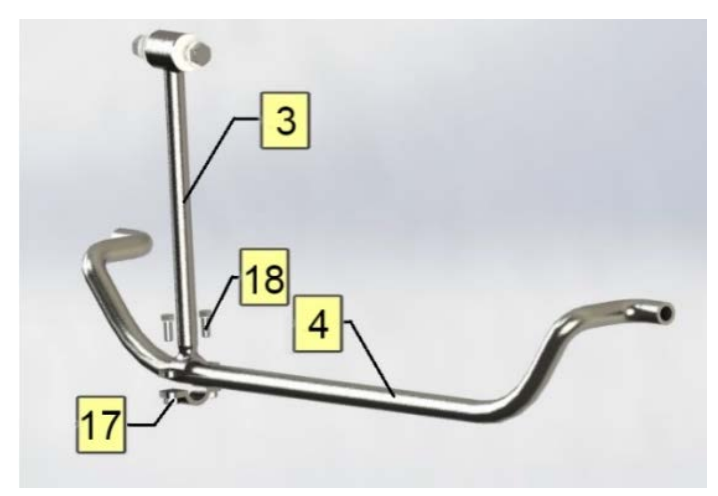

Fig. 3: Securing the stabilizer stand: 4) Stabilizer; 3) Stand; 17) Flange and 18) Bolts

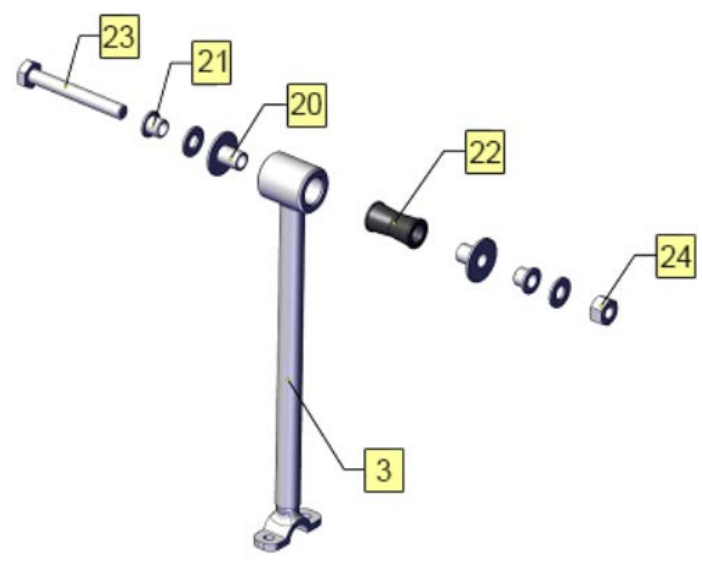

Fig. 4: Design of the silent block: 3) Stand; 20) Guide; 21) Bushing; 22) Rubber insert; 23) Bolt and 24) Nut

The hydraulic cylinder 2 is attached to the stabilizer 4 by means of a flange 25 andto the car body by means of a silent block 19. Fastening of hoses is carried out by the fitting 26 (Fig. 5).

The hydraulic cylinder 2 consists of: the housing 27 , the piston 28 , the rod 29 , the guide sleeve 30 , the nut 31 , the nut 32 (Fig. 6).

The piston of the hydraulic cylinder 2, consisting of: piston 33 , two cuffs 34 , retaining rings 35 with which it is fixed on the rod 36 (Fig. 7).

The device works as follows. The solenoid valve 1, receiving signals from the ECU 7 or forcibly when the driver presses the button, turns on or off. When moving on a flat surface, the valve 1 is closed, the liquid is not able to move from one cavity to another, so, the cylinder rod 36 does not move while the car has good handling. When driving on the road, the ECU 7 sends a signal to the solenoid valve 1 whereby the core 12 under the influence of the electromagnetic field generated by the coil 10 is

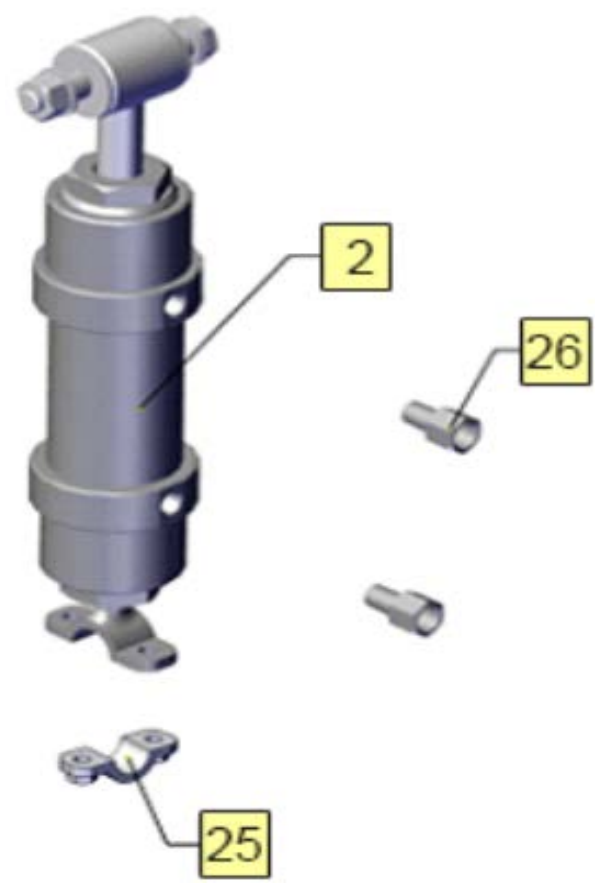

Fig. 5: Fitting attachment: 2) Hydraulic cylinder; 25) Flange and 26) Fitting

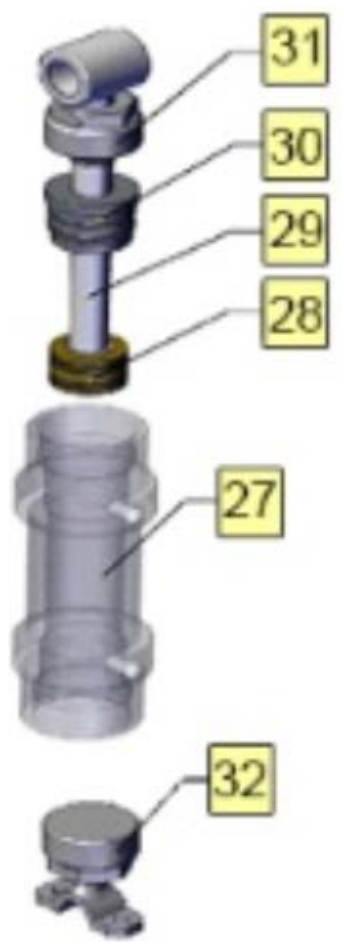

Fig. 6: Design of the hydraulic cylinder: 27) The housing; 28) Piston; 29) Stem; 30) Guide bushing; 31) Nut and 32) Nut 


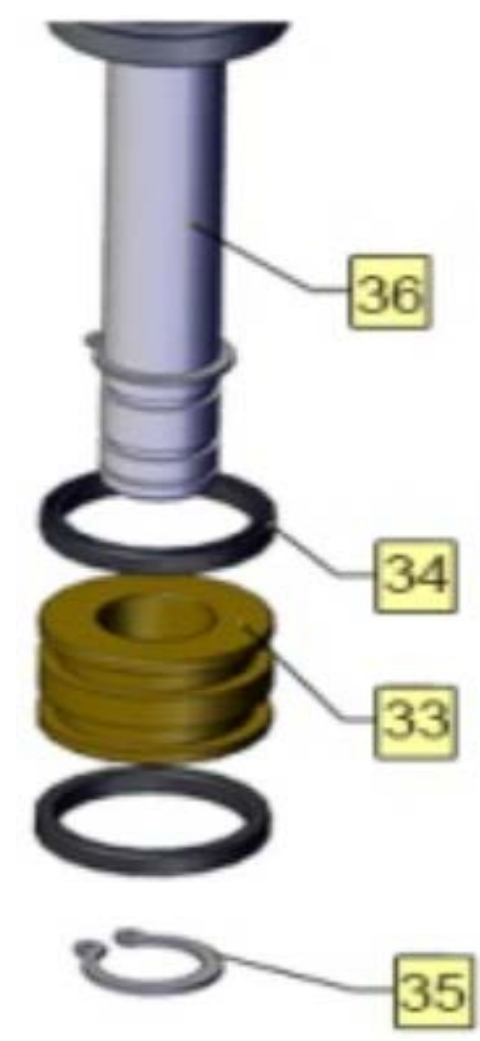

Fig. 7: Design of the piston: 33) Piston; 34) Cuff; 35) Retaining ring and 36) Stock

retracted, opening the channel in the head 13 , contributing to the free movement of fluid from the lower cavity of the cylinder 2 , through the hoses 5 through the valve 1 at the top and Vice versa. The rod 29 has the ability to move freely which increases the course of the levers and the energy intensity of the entire suspension, and consequently, the permeability. More suspension begins to absorb the energy of hitting the bump. The cover 9 of the solenoid valve 2 is attached to the body 16 by means of four nuts 8 . Together with them, the head 13 of the electromagnetic valve 2 is attached. The gasket 14 and the sealing ring 15 are used for tightness of connections.

The introduction of the proposed stabilization system will improve safety when driving a vehicle. Thus, the study proposes to expand the range of possibilities of the vehicle (Generalova and Khabibullin, 2018).

Optimization of the proposed design: The next stage of any development is to optimize the design solution. In the practice of designing products for automobile and tractor construction, parametric optimization systems are widely used which are a mandatory attribute of almost all systems of packages for mathematical modeling of objects. At the same time in some cases it is easier to

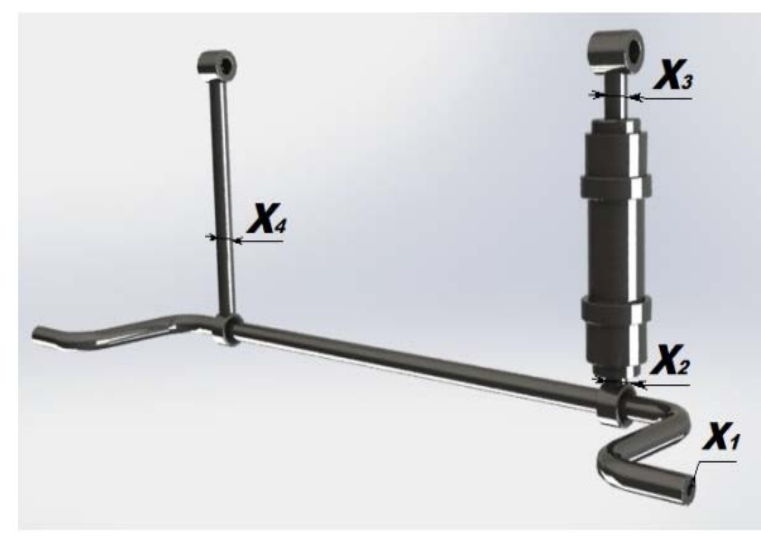

Fig. 8: Varying parameters

create your own system that provides the ability to solve similar problems on the fly. Usually, this is done using brute force methods or alternating-variable descent method with learning (D'yachkov et al., 2016a, b, 2018).

One of the first steps in optimizing the design is to select variable parameters. As the main parameters responsible for the performance of the proposed system, the thickness of the stabilizer of transverse stability, the diameter of the stabilizer bar, the diameter of the hydraulic cylinder rod and the diameter of the hydraulic cylinder rack are taken. These design parameters are responsible for the following characteristics: safety margin $S$, stress $\sigma$, fatigue $U$ and mass $\mathrm{m}$. The heterogeneity of the design parameters is due to different modes of force from the road irregularities. Determination of the optimal values of these parameters will allow to develop a reliable and non-metal-intensive design (Fig. 8).

\section{Optimization options:}

- $\mathrm{X}_{1}$-thickness of the stabilizer $(\mathrm{mm})(9, \ldots, 14)$

- $\mathrm{X}_{2}$-diameter of the hydraulic cylinder stand $(\mathrm{mm})$ $(20, \ldots, 28)$

- $\mathrm{X}_{3}$-diameter of the hydraulic cylinder $\operatorname{rod}(\mathrm{mm})(20, \ldots$, 28)

- $\quad \mathrm{X}_{4}$-stabilizer stand diameter $(\mathrm{mm})(20, \ldots, 28)$

In such situations, experimental plans with a stable (regular) structure regardless of the number of variable parameters are preferable. This makes it possible to form such plans in an automated mode as well as to build a regression model of the process and optimize on its basis a set of parameters and characteristics of the processes. The regularity of the structures has for example, the plans of the full factorial experiment (the number of parameters 
is not limited), the plans of the second order of the Rechtshafner (the number of parameters is not limited), the box-draper (with the number of parameters from 2-15), plans based on hyper-greek-latin squares (the number of parameters is not limited). The number of minimum required experiments is significantly reduced by the index of these plans.

The study considers the procedure of constructing polynomial models of second order on the basis of the plan of rechtshafner. The solution is implemented in MathCad system. To solve the problem, an algorithm with MathCad system is implemented which contains the following procedures:

- Formation of the plan and the expanded matrix of planning

- Export settings and import values of the characteristics obtained in the module of SolidWorks

- Calculation of the values of the coefficients of the regression equation

- Formation of the values of the initial calculation points in the range $-1, \ldots,+1$

- Translation of the coded values of the optimized parameters into real values

- Translation of real values of optimized parameters into coded values

- Calculation of the value of the private quality criterion in the nodes of the plan

- Search of optimum (use the method of complete enumeration)
- Determination of optimal values of parameters in coded and natural views

- Control the order of calculation and decoding parameters

The extended planning matrix W1 for task conditions is shown in Fig. 9, columns 6 through 9 are elements of the planning matrix.

In most technical applications, the optimum domain is approximated fairly accurately by second-degree polynomials. To solve this problem, the polynomial of four variables has the form (Eq. 1):

$$
\begin{aligned}
& \text { Polinom }(\mathrm{B}, \mathrm{x} 1, \mathrm{x} 2, \mathrm{x} 3, \mathrm{x} 4)=\mathrm{B} \_15+\mathrm{B} \_1 \mathrm{x} 1+ \\
& \mathrm{B} \_2 \times 2+\mathrm{B}_{-} 3 \times 3+\mathrm{B}_{-} 4 \times 4+\mathrm{B} \_5 \times 1 \times 1+ \\
& \mathrm{B} \_6 \times 1 \times 2+B_{-} 7 \times 1 \times 3+B_{-} 8 \times 1 \times 4+ \\
& \text { B_9x } 2 \times 2+B \_10 \times 2 \times 3+B \_11 \times 2 \times 4+ \\
& \text { B_ } 12 \times 3 \times 3+B \_13 \times 3 \times 4+B \_14 \times 4 \times 4
\end{aligned}
$$

Based on the experimental data obtained, a mathematical model (Eq. 2) of the stabilization system consisting of four regression equations is constructed. This model describes the nature of loading of the proposed stabilization system in the given initial conditions:

$$
\left\{\begin{array}{l}
\mathrm{S}=\text { Polinom }(\mathrm{BS}, \mathrm{x} 1, \mathrm{x} 2, \mathrm{x} 3, \mathrm{x} 4) \rightarrow \max \\
\sigma=\operatorname{Polinom}(\mathrm{B} \sigma, \mathrm{x} 1, \mathrm{x} 2, \mathrm{x} 3, \mathrm{x} 4) \rightarrow \min \\
\mathrm{U}=\operatorname{Polinom}(\mathrm{BU}, \mathrm{x} 1, \mathrm{x} 2, \mathrm{x} 3, \mathrm{x} 4) \rightarrow \max \\
\mathrm{m}=\text { Polinom }(\mathrm{Bm}, \mathrm{x} 1, \mathrm{x} 2, \mathrm{x} 3, \mathrm{x} 4) \rightarrow \min
\end{array}\right.
$$

\begin{tabular}{|r|r|r|r|r|r|r|r|r|r|r|r|r|r|r|r|}
\hline & 1 & 2 & 3 & 4 & 5 & 6 & 7 & 8 & 9 & 10 & 11 & 12 & 13 & 14 & 15 \\
\hline 1 & 1 & 1 & 1 & 1 & 1 & -1 & -1 & -1 & -1 & 1 & 1 & 1 & 1 & 1 & 1 \\
\hline 2 & 1 & 1 & 1 & 1 & 1 & -1 & 1 & 1 & 1 & -1 & -1 & -1 & 1 & 1 & 1 \\
\hline 3 & 1 & 1 & 1 & 1 & 1 & 1 & -1 & 1 & 1 & -1 & 1 & 1 & -1 & -1 & 1 \\
\hline 4 & 1 & 1 & 1 & 1 & 1 & 1 & 1 & -1 & 1 & 1 & -1 & 1 & -1 & 1 & -1 \\
\hline 5 & 1 & 1 & 1 & 1 & 1 & 1 & 1 & 1 & -1 & 1 & 1 & -1 & 1 & -1 & -1 \\
\hline 6 & 1 & 1 & 1 & 1 & 1 & 1 & 1 & -1 & -1 & 1 & -1 & -1 & -1 & -1 & 1 \\
\hline 7 & 1 & 1 & 1 & 1 & 1 & 1 & -1 & 1 & -1 & -1 & 1 & -1 & -1 & 1 & -1 \\
\hline 8 & 1 & 1 & 1 & 1 & 1 & 1 & -1 & -1 & 1 & -1 & -1 & 1 & 1 & -1 & -1 \\
\hline 9 & 1 & 1 & 1 & 1 & 1 & -1 & 1 & 1 & -1 & -1 & -1 & 1 & 1 & -1 & -1 \\
\hline 10 & 1 & 1 & 1 & 1 & 1 & -1 & 1 & -1 & 1 & -1 & 1 & -1 & -1 & 1 & -1 \\
\hline 11 & 1 & 1 & 1 & 1 & 1 & -1 & -1 & 1 & 1 & 1 & -1 & -1 & -1 & -1 & 1 \\
\hline 12 & 1 & 1 & 0 & 0 & 0 & 1 & 0 & 0 & 0 & 0 & 0 & 0 & 0 & 0 & 0 \\
\hline 13 & 1 & 0 & 1 & 0 & 0 & 0 & 1 & 0 & 0 & 0 & 0 & 0 & 0 & 0 & 0 \\
\hline 14 & 1 & 0 & 0 & 1 & 0 & 0 & 0 & 1 & 0 & 0 & 0 & 0 & 0 & 0 & 0 \\
\hline 15 & 1 & 0 & 0 & 0 & 1 & 0 & 0 & 0 & 1 & 0 & 0 & 0 & 0 & 0 & 0 \\
\hline
\end{tabular}

Fig. 9: Advanced planning matrix 
Table 1: The values of the coefficients of the regression equation

\begin{tabular}{lcccc}
\hline Coefficients & Margin of safety (BS) & Von mises stresses, B $\sigma(\mathrm{MPa})$ & Fatigue BU, loading cycles & Mass Bm (kg) \\
\hline $\mathrm{B}_{15}$ & -0.028 & -129.500 & $5.153 \cdot 10^{5}$ & 7.505 \\
$\mathrm{~B}_{1}$ & -0.479 & -108.364 & $-1.015 \cdot 10^{6}$ & -2.666 \\
$\mathrm{~B}_{2}$ & 0.478 & -116.601 & $9.368 \cdot 10^{5}$ & -2.630 \\
$\mathrm{~B}_{3}$ & -0.225 & 38.772 & $-2.464 \cdot 10^{5}$ & -2.592 \\
$\mathrm{~B}_{4}$ & $-4 \cdot 10^{3}$ & 1.646 & $-1.16 \cdot 10^{4}$ & -0.319 \\
$\mathrm{~B}_{5}$ & $2.5 \cdot 10^{3}$ & 2.759 & $-3.425 \cdot 10^{3}$ & $1.167 \cdot 10^{-3}$ \\
$\mathrm{~B}_{6}$ & $2.5 \cdot 10^{3}$ & 2.947 & $-4.275 \cdot 10^{3}$ & $-8.333 \cdot 10^{5}$ \\
$\mathrm{~B}_{7}$ & 0 & -1.598 & $-2.175 \cdot 10^{3}$ & $-8.333 \cdot 10^{5}$ \\
$\mathrm{~B}_{8}$ & $1.563 \cdot 10^{3}$ & 2.079 & $1.731 \cdot 10^{4}$ & 0.055 \\
$\mathrm{~B}_{9}$ & 0.02 & -0.374 & $5.484 \cdot 10^{3}$ & $-5.208 \cdot 10^{5}$ \\
$\mathrm{~B}_{10}$ & 0 & -0.976 & $5.047 \cdot 10^{3}$ & $1.042 \cdot 10^{-4}$ \\
$\mathrm{~B}_{11}$ & -0.027 & 2.468 & $-1.942 \cdot 10^{4}$ & 0.056 \\
$\mathrm{~B}_{12}$ & 0 & -0.897 & $-2.281 \cdot 10^{3}$ & $-5.208 \cdot 10^{5}$ \\
$\mathrm{~B}_{13}$ & 0.588 & $3.891 \cdot 10^{3}$ & 0.056 \\
$\mathrm{~B}_{14}$ & $4.688 \cdot 10^{3}$ & -0.324 & $7.044 \cdot 10^{5}$ & 65.767 \\
\hline
\end{tabular}

The obtained coefficients of the regression equations are summarized in Table 1. Multi-mode operation of the product leads to the need to form a compromise solution that best meets the totality of conflicting requirements for the designed product (Podinovsij and Nogin, 1982). For this reason, it is necessary to use the integral criterion of quality of the project excluding possibility of compensation of loss of quality of one indicators by a high indicator of other indicators. Its use should be based on the Pareto principle which provides the possibility of reducing the problem with a set of individual criteria to the task with a single integrated criterion of quality. At the same time, the values of the weights of the particular criteria can be determined and refined automatically in the course of solving the problem.

The determination of the system of weight coefficients and the ranking of Pareto solutions on it leads to an optimal compromise balanced by the inconsistency of the particular criteria. The integral quality criterion $\mathrm{SA}_{i}$ can be represented as follows (Podinovsij and Nogin, 1982):

$$
\begin{aligned}
& \text { if } \varphi_{i} \rightarrow \min , \quad \text { then } \mathrm{SA}_{\mathrm{i}}=\left(\sum \omega_{1}^{2}\left(\frac{\varphi_{\mathrm{i}}}{\varphi_{\mathrm{imin}}}-1\right)^{2}\right)^{\frac{1}{2}} \\
& \text { if } \varphi_{\mathrm{i}} \rightarrow \max , \text { then } \mathrm{SA}_{\mathrm{i}}=\left(\sum \omega_{1}^{2}\left(\frac{\varphi_{\mathrm{imax}}}{\varphi_{\mathrm{i}}}-1\right)^{2}\right)^{\frac{1}{2}}
\end{aligned}
$$

Here, $\varphi$ and $\omega$ are particular quality criteria and their "weight" values. The quadratic form of the record provides a "deflection" the point of compromise project. The weight coefficients are determined and refined in the course of solving the problem automatically by successive narrowing of the range of variation of the required parameters near the optimum point (deflection), since the "weights" are a function of the width of the variation area. For this problem all weights are equal and equal (Eq. 3).

$$
\mathrm{SA}_{\mathrm{i}}=\left[\begin{array}{l}
0.25^{2}\left(\frac{\mathrm{S}_{\mathrm{i}}}{\mathrm{S}_{\mathrm{i} \text { min }}}-1\right)^{2}+0.25^{2}\left(\frac{\frac{1}{\sigma_{\mathrm{i}}}}{\sigma_{\mathrm{i} \min }}-1\right)^{2}+ \\
+0.25^{2}\left(\frac{\mathrm{U}_{\mathrm{i}}}{\mathrm{U}_{\mathrm{i} \min }}-1\right)^{2}+0.25^{2}\left(\frac{\frac{1}{\mathrm{~m}_{\mathrm{i}}}}{\mathrm{m}_{\mathrm{i} \min }}-1\right)^{0.5}
\end{array}\right]^{2}
$$

For the automated mode, the significance of the regression equation coefficients is not checked. For further calculations are used not physical quantities $\mathrm{S}, \sigma$, $\mathrm{U}, \mathrm{m}$ and the corresponding dimensionless $\mathrm{S} 1, \sigma 1$, $\mathrm{U} 1, \mathrm{~m} 1$.

Figure 10 shows graphs of the dependence of the dimensionless values of the partial quality criteria $\mathrm{S} 1, \sigma 1$, $\mathrm{U} 1, \mathrm{~m} 1$ on the iteration number i: S1(i) safety margin graph, $\sigma 1$ (i) von Mises stress graph, U1(i) fatigue graph, m1(i) mass graph.

Figure 11 shows the multi-extremal nature of the integral quality criterion $\mathrm{SA}_{\mathrm{i}}$. Therefore, to search for a global extremum, the full search algorithm implemented in MathCad is used. The following values are obtained by partial optimization of the proposed stabilization system:

- $\mathrm{X}_{1}=13.167$-thickness of stabilizer (mm)

- $\mathrm{X}_{2}=20$-diameter of the hydraulic cylinder rack (mm)

- $\mathrm{X}_{3}=28$-diameter of the cylinder $\operatorname{rod}(\mathrm{mm})$

- $\mathrm{X}_{4}=20$-diameter of the stabilizer stand (mm)

In the SolidWorks system, an experiment was conducted with the set values of design parameters. The calculation error was $<7 \%$.

Since, the quality criterion is calculated in 4-dimensional space, the number of mutually orthogonal pairs of axes is 6 (these are the following pairs $\mathrm{XY}, \mathrm{XZ}$, 


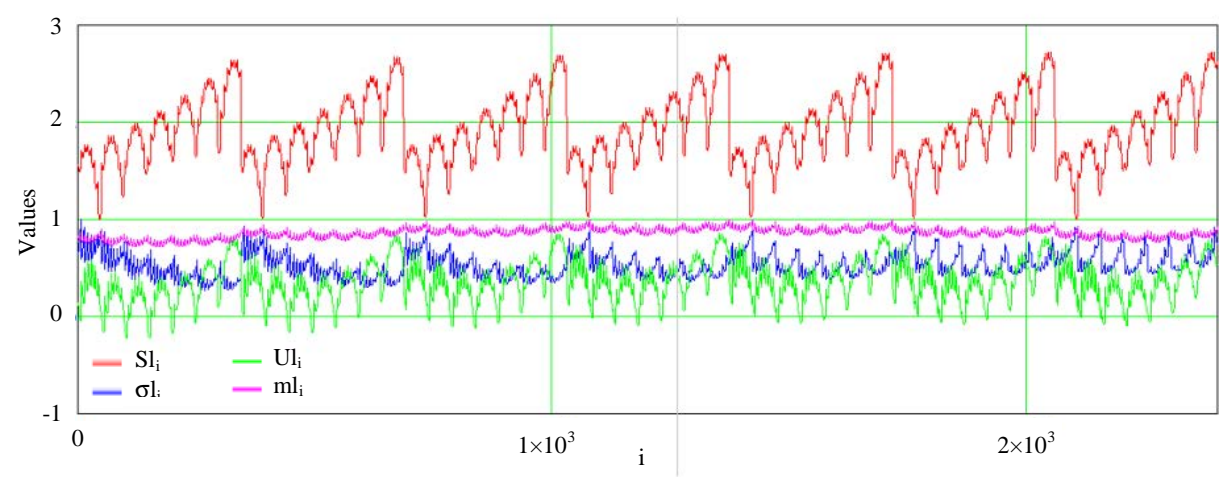

Fig. 10: Graphs of dimensionless values of partial quality criteria

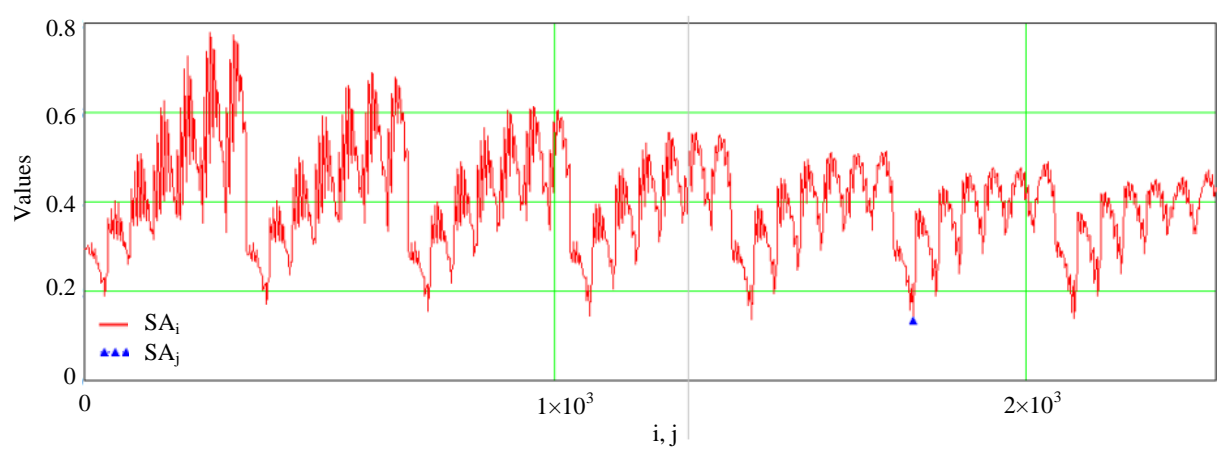

Fig. 11: Graph of the values of the integral criterion of quality

$\mathrm{XT}, \mathrm{YZ}, \mathrm{YT}, \mathrm{ZT}$ ). In substitution instead of X Y Z and $\mathrm{T}$, the necessary parameters, on which the quality criterion depends are obtained projections on the corresponding planes. This technique allows us to consider a four-dimensional model that is to understand more deeply how the quality criterion behaves in this case. It should be noted that such an approach can be applied when the number of factors is not more than 5 because otherwise the computational process will be time-consuming.

This approach can be applied in cases where the design or the study of performance there is a problem of using not the absolute optimum of the integral quality criterion and it's about the optimal value. For example, the definition of close to the optimum values will allow to find more favorable dimensions of the stabilization system in terms of maintainability interchangeability, ergonomics, aesthetics or other indicator.

In this study, a deviation of $10 \%$ from the absolute optimum is accepted for calculation near the optimal value of the integral criterion. Since, the quality criterion depends on 4 independent parameters that is their orthogonality is implied, four independent cycles of search by ranked variables were formed. The calculation results are shown in Fig. 12.
Figure 12 shows 6 mutually perpendicular planes intersecting the set of 4-dimensional solutions of the integral quality criterion. The points of all found solutions obtained by a full search are indicated in red andthe values of characteristics near the optimal solutions are indicated in blue (the values have the index sp). The obtained graphs allow us to estimate the vastness of variation by the criteria.

Thus, you can find not one solution but several. These solutions will be approximately the same in optimality but can vary greatly in size. The resulting spectra of the resulting characteristics can determine the optimized product within which it is possible to vary the characteristics.

Also for the obtained about optimal solutions in this study, a range of factors that can be varied, staying close to the optimum which can allow you to choose more convenient desired parameters.

In Fig. 13, the red lines indicate the upper and lower boundaries (the formed central rectangle is the search area, it is divided into 6 segments for each factor and forms a solution field of $7 \times 7$ points), blue points are one optimal solution corresponding to the minimum value of the quality criterion SA, blue non-shaded points are near-optimal solutions. 

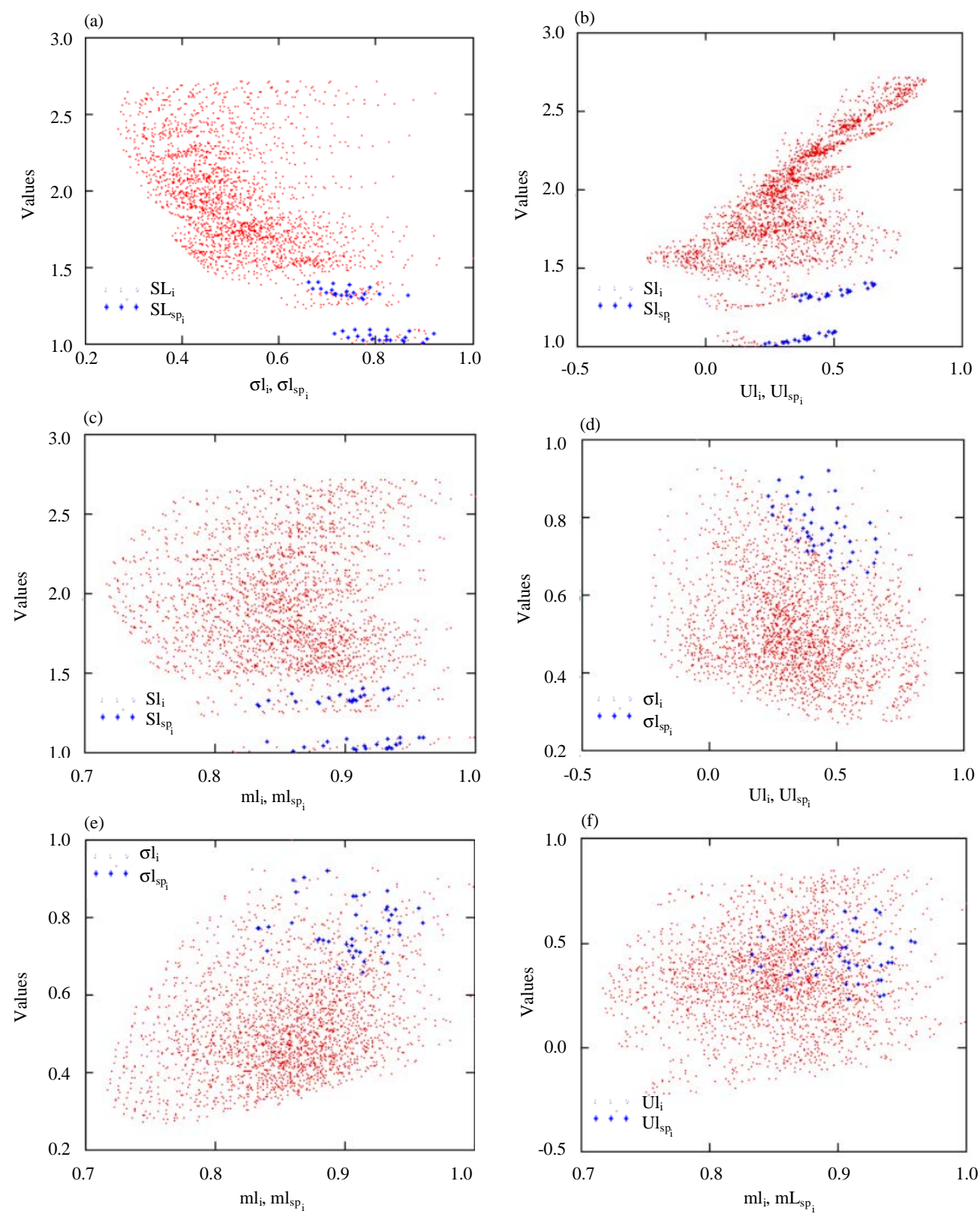

Fig. 12: a-f) Graphs of the spectrum of the optimal values of the experimental results corresponding to the region of the minimum values of the quality criterion SA

As can be seen from the graphs, not all factors affect the solution to the same extent for example in the field of factors $\mathrm{X}_{1}$ and $\mathrm{X}_{4}$, it is possible to vary the parameters sufficiently without significant damage to the optimal solution.

In the SolidWorks environment an experiment was performed with the design parameter values set, the results of which are shown in Fig. 14. The calculation error was $<7 \%$.
The obtained parameter values can be used to clarify the suspension structure and the control principles of its characteristics, taking into account the list of the operating modes.

The considered approach to the optimization of the design process parameters and characteristics of the stabilization system really provides an opportunity to expand the classes of problems solved by developers within a single information and time space is a specific 

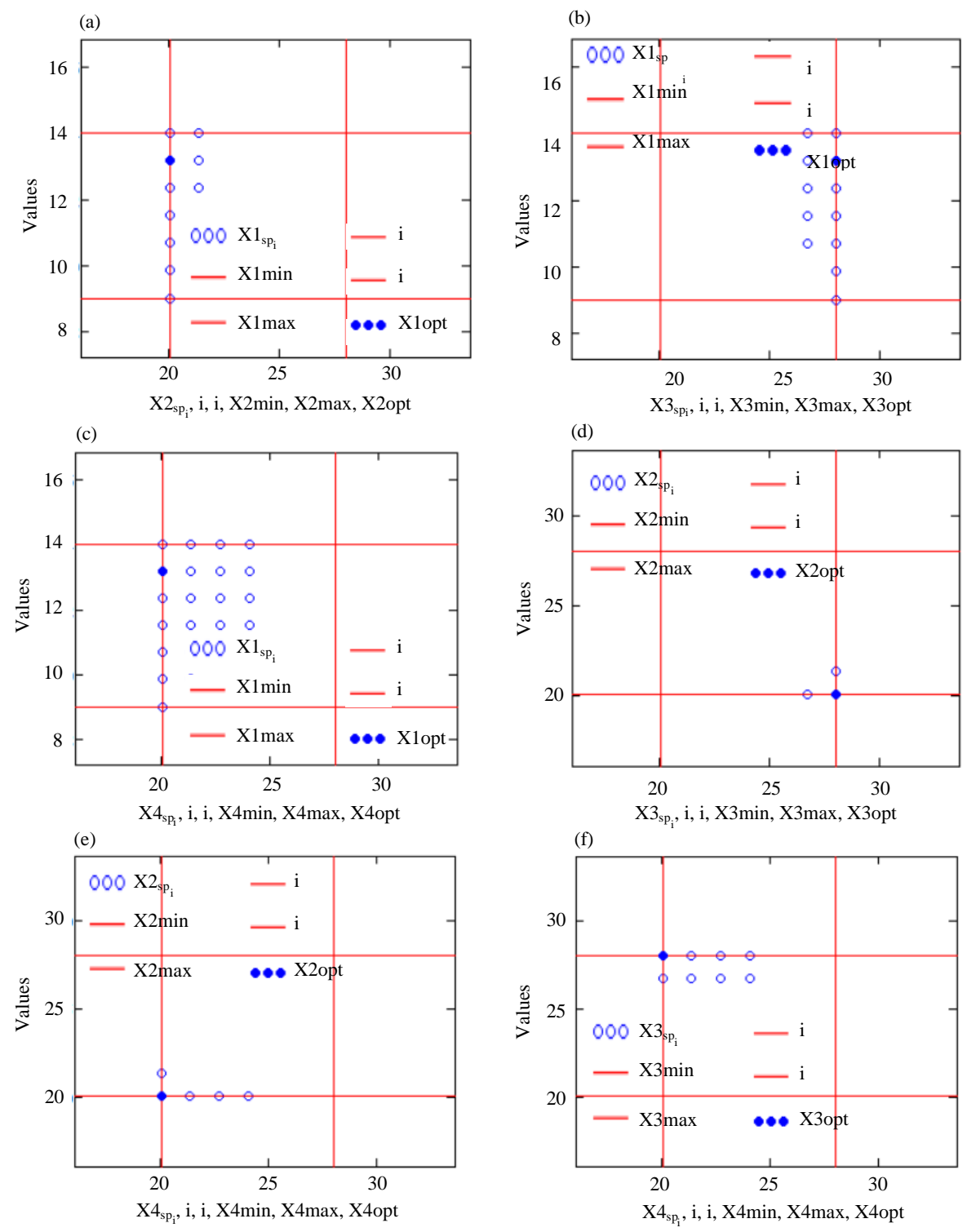

Fig. 13: a-f) Graphs of the spectra of the optimal values of the factors corresponding to the region of the minimum values of the quality criterion SA

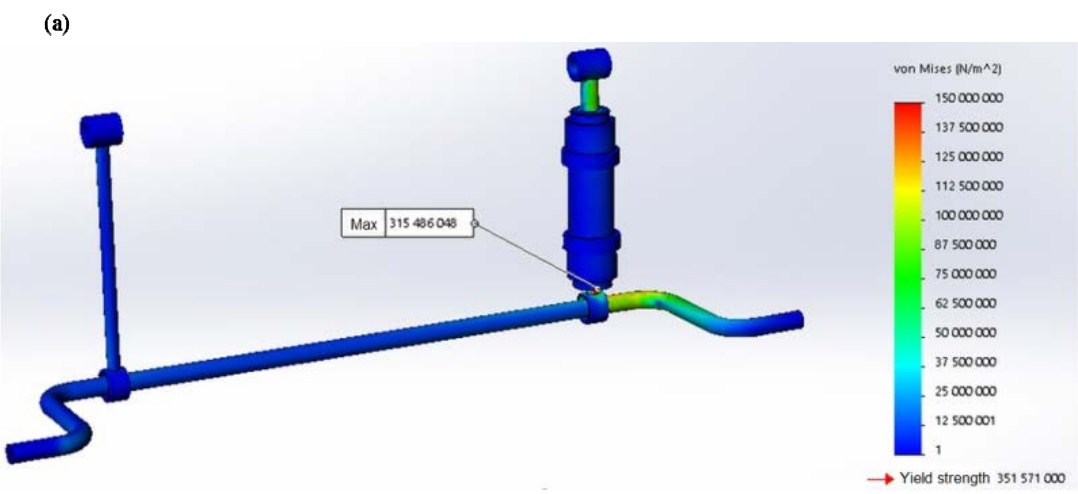

Fig. 14: Continue 
(b)
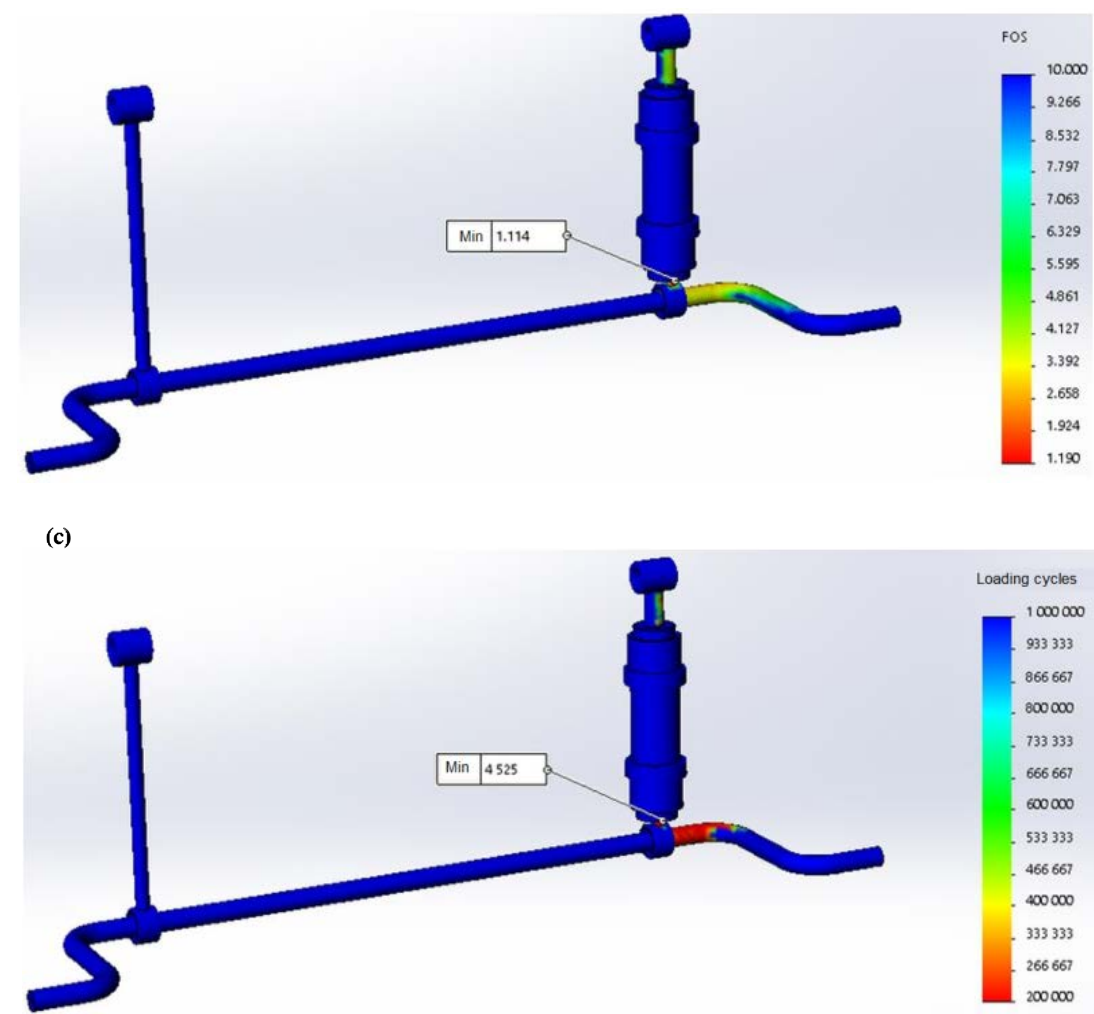

Fig. 14: a) Stress diagrams; b) Safety factor and c) Fatigue

tool to ensure quality in accordance with a set of conflicting requirements for the conditions of operation.

\section{CONCLUSION}

Analysis of the results allows us to draw the following conclusions: the system of stabilization of the car which is implemented in the design of electromagnetic control, allowing to exclude from the design of the stabilization system additional connecting hoses and drives and improve the safety of driving. An approach for determining the optimal value of structural elements which allows to ensure the quality of the proposed stabilization system in accordance with a set of conflicting requirements for the operating conditions is proposed. The weight and size characteristics for the proposed stabilization system are determined. The calculation error in the simulation was $<7 \%$. A mathematical apparatus for determining the range of optimal values of the proposed design, allowing you to select more convenient desired parameters, unlike the absolute optimum of not more than $10 \%$.

\section{ACKNOWLEDGEMENTS}

This design of the continuously variable transmission was developed at the Department of
Transport Machines of Penza State University. The presented took 1st place at the IV all-Russian scientific and technical conference "Innovations of technical solutions in mechanical engineering and transport" Penza (2018). Also, the novelty and efficiency of the proposed design is confirmed by the patent of the Russian Federation No. 2675681 (Generalova and KHabibullin, 2018).

\section{REFERENCES}

Bogatyryov, A.V., Y.U.K. Esenovskij-Lashkov, M.L. Nasonovskij and V.A. CHernyshev, 2004. Avtomobili [Vehicle]. KolosS, Moscow, Russia,

Chun, H.H., S.J. Kwon and T. Tak, 2007. Reliability-based design optimization of automotive suspension systems. Intl. J. Automot. Technol., 8: 713-722.

D'yachkov, Y.U.A., A.A. Semyonov and A.A. Generalova, 2016a. [Applied Optimization in the Design of Wheeled Vehicles Studies Benefit]. Mir Nauki, Moscow, Russia, ISBN:978-5-9908912-0-3, Pages: 210 (In Russian).

D'yachkov, Y.U.A., A.A. Semyonov and A.A. Generalova, 2016b. [Optimization of Structures of Land Transport and Technological Means Studies Benefit]. Mir Nauki, Moscow, Russia, (In Russian). 
D'yachkov, Y.U.A., I.P. Toropcev and A.A. Generalova, 2018. [Modeling of Systems in Automobile and Tractor Construction Studies Benefit]. Mir Nauki, Moscow, Russia,ISBN:978-5-6041425-1-6,Pages: 115 (In Russian).

Generalova, A.A. and R.R. KHabibullin, 2018. [Dynamic stabilization of the car body]. Master Thesis, Federal State Budgetary Educational Institution of Higher Education, Penza State University, Penza, Russia. (In Russian)

Pavlov, V.V. and V.V. Kuvshinov, 2011. [Theory of Motion of Multi-Purpose Tracked and Wheeled Vehicles]. Cheboksary Printing House, Cheboksary, Russia, (In Russian).
Pavlov, V.V., 2014. [Design Calculations of SpecialPurpose Vehicles: Studies Benefit]. Moscow Automobile and Road Construction University, Moscow, Russia, (In Russian).

Podinovsij, V.V. and V.D. Nogin, 1982. [Pareto-Optimal Solutions of Multicriteria Problems]. Nauka, Moscow, Russia, (In Russian).

Sidel'nikov, I.D. and A.E. Brom, 2018. Optimization of diversified stock for military and special purpose equipment under specified failure conditions. Sci. Perspect., 3: 15-19.

Suh, K. and H. Yoon, 2018. Design optimization of a rear independent suspension for the Korean light tactical vehicle. Intl. J. Automot. Technol., 19: 245-252. 